RICYDE. Revista Internacional de Ciencias del Deporte doi: $10.5232 /$ ricyde

Rev. int. cienc. deporte

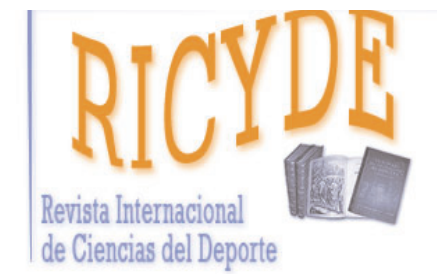

RICYDE. Revista Internacional de Ciencias del Deporte VOLUMEN XI - ANO XI

Páginas:329-338 ISSN:1885-3137

Número 42 - Octubre - 2015

\title{
Autopercepción de inteligencia contextual para jugar y de competencia decisional en el fútbol \\ Game intelligence and perceived competence to decide on soccer players
}

\author{
Luis Miguel Ruiz-Pérez ${ }^{1}$, José Antonio Navia ${ }^{2}$, Javier Miñano-Espín ${ }^{1}$, \\ Virginia García-Coll ${ }^{3}$, Miriam Palomo-Nieto 4
}

1.Universidad Politécnica de Madrid. España 2.Universidad Pontificia de Salamanca. España 3.Universidad Internacional de La Rioja. España

4.Universidad Palacky. Olomouc. Czech Republic

\section{Resumen}

En el presente estudio se analizó la autopercepción de competencia decisional y la inteligencia contextual de jugadores de fútbol de diferente nivel de pericia. Además, se exploraron las relaciones existentes entre las autopercepciones de competencia para decidir y de inteligencia contextual en jugadores de fútbol, para lo cual participaron voluntariamente 467 jugadores $(M=20,26, D T=4,83)$. El nivel de pericia de los participantes se estableció en tres diferentes: Autonómico $(N=141)$, nacional $(N=253)$ e internacional ( $N=73$ ), de un total de 46 clubes españoles así como de varias selecciones nacionales. Se utilizaron dos instrumentos en forma de cuestionarios. El primero fue el Cuestionario de Inteligencia Contextual Percibida en el Deporte (ICD) y el segundo el de Estilos de Toma de Decisión en el Deporte (CETD). Los resultados mostraron que la competencia decisional y la inteligencia contextual están relacionadas y mejoran según aumenta el nivel deportivo.

Palabras clave: toma de decisiones; inteligencia práctica; fútbol; auto-percepciones; pericia.

\section{Abstract}

The aim of this paper was to analyze decisional competence and contextual intelligence in sport among different level of expertise football players. In addition, the relationship between self-perceptions of decisional competence and contextual intelligence was assessed. Participants were 467 football players $(M=20,26, D T=5,43)$. They were assigned three levels of expertise: Autonomic $(N=141)$, National $(N$ = 253) and International $(\mathrm{N}=73)$, from a group of 46 spanish clubs and several national teams. The Contextual Intelligence in Sport Questionnaire (ICD) and the Decision Making in Sport Questionnaire (CETD) were used to explore self-perceptions of players. Results showed that self-perceptions of Contextual Intelligence and Decision Making increased with the level of expertise.

Key words: decision making; practical intelligence; soccer; self-perceptions; expertise. 
Ruiz-Pérez, L. M.; Navia, J. A.; Miñano-Espín, J.; Garcia-Coll, V. y Palomo-Nieto, M. (2015). Autopercepción de inteligencia contextual para jugar y de competencia decisional en el fútbol. RICYDE. Revista internacional de ciencias del deporte, 42(11), 329-338. http://dx.doi.org/10.5232/ricyde2015.04202

\section{Introducción}

$\mathrm{C}$ ada vez se conoce más sobre las cualidades que caracterizan a los deportistas de diferente nivel de pericia, sobre su forma de acometer los retos de su deporte, sus cualidades psicológicas y perceptivo-cognitivas (Ruiz, Duran, Jiménez y Sánchez, 2006; Ruiz, Durán y Arruza, 2007). El estudio de las autopercepciones ha sido una forma de analizar los estados, disposiciones y actitudes desde el punto de vista de los protagonistas, ya que como indicara Tenenbaum (2009), a pesar de su susceptibilidad a la deseabilidad social y otras fuentes de error, la fiabilidad y la validez de estos instrumentos han favorecido su empleo.

En este sentido, la literatura científica destaca cómo la inteligencia es un importante factor individual que predice el éxito en el trabajo (Drasgow, 2003). La dificultad reside en la propia definición de este constructo. En la actualidad existe un creciente interés por destacar el papel de otro tipo inteligencias diferentes a las evaluadas con el Coeficiente Intelectual (CI), ya que se sugiere por los investigadores la existencia de otros aspectos que pueden estar influyendo en el rendimiento inteligente de las personas (Sternberg y col., 2000). Así, tomando como referencia la revisión llevada a cabo por Furnham (2005) son diferentes las inteligencias que han sido destacadas por autores como Sternberg, Gardner, Salovey o Goleman, y con denominaciones tales como: Analítica, Corporal, Creativa, Emocional, Práctica, Contextual, Musical, para los Negocios, Espiritual, Espacial, Matemática o Verbal. Todo ello muestra la complejidad de esta noción y la necesidad de analizarla en su relación con el contexto en el que las personas deben actuar (Gardner, 1983, 1987, 1993, 2001; Goleman, 1996, 2006; Goleman y Cherniss, 2001; Sternberg, 2000; Terenzini, 1993).

Las numerosas conversaciones llevadas a cabo con deportistas o entrenadores, el análisis de la literatura especializada, el estudio del rendimiento deportivo en contextos naturales y los estudios llevados a cabo anteriormente en el ámbito de la pericia deportiva (Durán, 2003; Jiménez, 2004; Navia, 2013; Navia y Ruiz, 2014; Peñaloza, 2007) confirman la necesidad de incoporar al estudio del rendimiento inteligente en el deporte a los propios protagonistas, (Jones 2014).

Para los entrenadores, este rendimiento inteligente se hace presente en expresiones tales como: tener criterio, carácter, visión de juego, anticipar, saber leer las jugadas, conocer el juego o dominar el contexto. En todos ellos destaca cómo la actuación inteligente en el deporte se hace presente en la capacidad para conocer la dinámica del juego, comprender las competencias que dichos contextos reclaman en cada momento, conocer sus propias competencias, conocer qué aspectos son críticos para alcanzar un objetivo y saber cómo lograr el éxito. Por todo ello parece adecuado hablar de una Inteligencia Práctica o Contextual en el Deporte (Ruiz, Graupera y García, 2014; Sternberg y col., 2000).

Los teóricos de la Inteligencia Contextual plantean que es la que se pone en acción cuando la persona tiene que adaptarse, seleccionar y acomodarse al entorno para obtener objetivos personalmente valiosos o el éxito en un ámbito de trabajo (Cianciolo, Matthew, Sternberg y Wargner, 2006). En el deporte sería la inteligencia reclamada para resolver problemas deportivos, y una de las diferencias principales que tienen los problemas deportivos respecto de los académicos, es que en el deporte la información necesaria para solucinarlos no suele estar presente en su totalidad, y su naturaleza cambiante y dinámica, los suele caracterizar como problemas mal definidos (Sternberg et al., 2000). Además, la inteligencia contextual deportiva reclama el empleo coordinado y preciso de movimientos corporales, lo cual nos aproximaría a la noción de Inteligencia Cinestésico Corporal de Gardner (1983). 
Ruiz-Pérez, L. M.; Navia, J. A.; Miñano-Espín, J.; Garcia-Coll, V. y Palomo-Nieto, M. (2015). Autopercepción de inteligencia contextual para jugar y de competencia decisional en el fútbol. RICYDE. Revista internacional de ciencias del deporte, 42(11), 329-338. http://dx.doi.org/10.5232/ricyde2015.04202

Por lo tanto, lo que reclama el deporte es una inteligencia dispuesta a solucionar problemas prácticos que implican movimientos precisos y controlados. Es la inteligencia del hacer, del llevar a cabo las soluciones, del know-how, del conocimiento tácito (Wagner, 1987), expresión de la pericia y excelencia de los deportistas, ya que reclama los conocimientos y habilidades necesarias para tener éxito en su deporte. Parafraseando a Sternberg (2000), estudiar la inteligencia contextual en el deporte se convierte en un método muy interesante para estudiar, comprender y analizar la pericia y cómo ésta se desarrolla.

Para los investigadores deportivos este aspecto no ha sido extraño y lo han considerado con expresiones diferentes, tales como sentido de juego (Game sense), visión de juego, conocimiento táctico o competencia táctica (Elferink-Gemser, Visscher, Richard y Lemmink, 2005; McPherson, 1994). Incluso el propio Coleman Griffith (1928) consideró que los jugadores de béisbol debían poseer lo que denominaba mental resolve, la capacidad de resolver los problemas del juego. Esta Inteligencia Contextual Deportiva supone el despliegue de cualidades tales como la iniciativa, la capacidad de decidir, la anticipación, la competitividad, la metacognición, la adaptabilidad, la rapidez mental o la planificación oportunista ante situaciones exigentes en lo psicológico, coordinativo y fisiológico, y que permitiría que el deportista pueda rendir con posibilidades de éxito.

A pesar de la importancia de este tipo de inteligencia en el desempeño deportivo, no abundan los estudios científicos que aborden este tema. Como excepciones, podemos encontrar los trabajos llevados a cabo por Ruiz y colaboradores (Ruiz, Graupera y García, 2014; Ruiz, García, Palomo, Navia y Miñano, 2014). La existencia de estos constructos que exploran la competencia de los deportistas para solventar los problemas que el juego les reclama, y el interés por conocer el grado de relación o independencia que ambos pudieran tener, hace que el presente estudio haya tenido como objetivo: a) analizar la inteligencia contextual y su relación con autopercepción de competencia decisional, considerando el efecto que el nivel de rendimiento pudiera tener en jugadores de fútbol españoles.

\section{Método}

\section{Participantes}

En el estudio participaron 467 jugadores de fútbol, todos ellos varones, de edades comprendidas entre los 14 y los 38 años $(M=20,26, D T=4,83)$. El dominio de los participantes se estableció en tres niveles diferentes según la categoría de sus equipos en: autonómico $(N=141)$, nacional $(N=253)$ e internacional $(N=73)$, de un total de 27 clubes españoles así como varios equipos y selecciones nacionales. Todos ellos aceptaron participar voluntariamente en este estudio tras firmar una hoja de consentimiento. En el caso de los menores de edad, se solicitó el permiso correspondiente a padres o tutores.

\section{Instrumentos}

Para recoger las autoperceptiones de competencia decisional entre los futbolistas se utilizó el Cuestionario de Estilos de Toma de Decisión en el Deporte - CETD (Ruiz, Graupera y Navarro, 1998; Ruiz, Graupera y Sánchez, 2000). Está compuesto por 30 ítems divididos en tres subescalas: Competencia Decisional Percibida (CDP), Ansiedad y Agobio al Decidir (AAD) y Compromiso en el Aprendizaje Decisional (CAD). Se presenta en una escala tipo Likert de 4 puntos, yendo del 1 (Totalmente en desacuerdo) al 4 (Totalmente de acuerdo). De las tres subescalas, la Competencia Decisional Percibida y Compromiso en el Aprendizaje Decisional son de carácter positivo, es decir, valores altos indicarían que las percepciones personales y el compromiso de los deportistas es elevado, mientras que la subescala de Ansiedad y Agobio al Decidir es de carácter negativo, de ahí que las puntuaciones altas en 
Ruiz-Pérez, L. M.; Navia, J. A.; Miñano-Espín, J.; Garcia-Coll, V. y Palomo-Nieto, M. (2015). Autopercepción de inteligencia contextual para jugar y de competencia decisional en el fútbol. RICYDE. Revista internacional de ciencias del deporte, 42(11), 329-338. http://dx.doi.org/10.5232/ricyde2015.04202

esta última subescala serían un indicador de desajuste emocional ante este tipo de situaciones. En cuanto a propiedades de medida, la consistencia interna (coeficiente de alpha de Cronbach) fue 0.82 para Competencia Decisional Percibida; de 0.83 para Ansiedad y Agobio al Decidir y de 0.75 para Compromiso en el Aprendizaje Decisional.

También se empleó el Cuestionario de Inteligencia Contextual Percibida en el deporte - ICD (Ruiz, Graupera, y García, 2014) Consta de 24 ítems y tres subescalas: Inteligencia Anticipatoria (IA), Inteligencia Táctica (IT) e Inteligencia Competitiva (IC). Se presenta en una escala tipo Likert de 10 puntos, yendo del 1 (Totalmente en desacuerdo) al 10 (Totalmente de acuerdo). Con ello se obtiene una medida global de Inteligencia Contextual en el Deporte (ICD). Este instrumento posee las cualidades de medida necesarias para su empleo en contextos diferentes, incluido el contexto investigador, siendo su fiabilidad de .90 para la subescala de Inteligencia Anticipatoria, de .87 para la Inteligencia Táctica y de .84 para la Inteligencia Competitiva.

\section{Diseño}

El diseño general de este estudio se encuentra incardinado en lo que se denomina paradigma asociativo (Arnau, 1995). En este tipo de sistema metodológico del diseño deriva de objetivos de covariación formulados con enunciados relacionales, de ahí que el diseño de esta investigación sea comparativo y diferencial, porque busca establecer las diferencias existentes entre grupos muestrales (que asumen el papel de variables independientes) en las variables dependientes. Las variables dependientes de esta investigación fueron los factores (subescalas) de los dos instrumentos empleados. Las variables independientes de carácter selectivo, fueron los grupos de nivel deportivo.

\section{Análisis estadísticos}

Los datos se analizaron descriptiva y diferencialmente. Para establecer los contrastes de diferencias de medias entre los grupos de nivel se realizaron Análisis Multivariados (MANCOVA) considerando como covariables los años de experiencia y los años compitiendo. Asimismo, se analizaron las relaciones entre las diferentes dimensiones de los dos instrumentos mediante análisis de correlación de Pearson. Para la valoración de los resultados se tuvo en cuenta generalmente el nivel de significación bilateral de .05. Además, se consideró conveniente informar del tamaño del efecto obtenido. Los cálculos estadísticos se realizaron con el programa SPSS 20.0.

\section{Resultados}

\section{Diferencias en función del nivel de los participantes}

En la Figura 1 se representan los datos descriptivos para las escalas del Cuestionario de Estilos de Toma de Decisiones y del Cuestionario de Inteligencia Contextual en el Deporte. En el contraste multivariado se encontró un efecto del nivel deportivo sobre el conjunto de las variables dependientes, $F_{(12,898)}=1.94, p=.027, \eta^{2}=.03$. Como muestra la Figura 1 izquierda, las diferencias de nivel se produjeron en la Competecia Decisional Percibida, $(F$ $(2,459)=3.63, p=.027 ; \eta^{2}=.016$, y en Ansiedad y Agobio al Decidir $(F(2,459)=5.18, p=.006$; $\left.\eta^{2}=.022\right)$. Aunque la tendencia encontrada fue que a medida que el nivel deportivo aumentaba así lo hacían las tres escalas del ICD, no se encontraron diferencias significativas en función del nivel de pericia para el resto de las variables (Figura 1 derecha). 

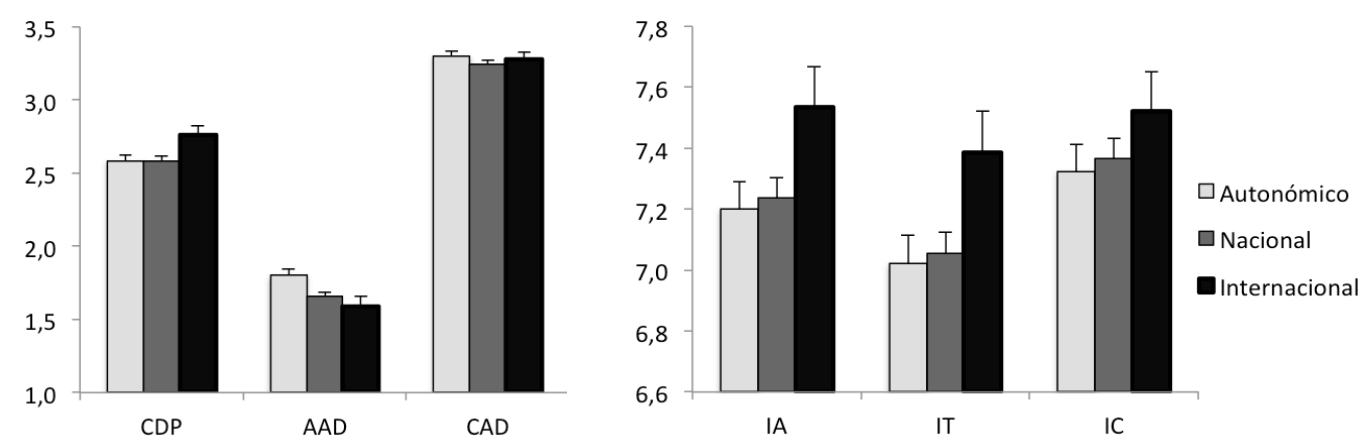

Figura 1. Puntuaciones obtenidas en las escalas del CETD (izquierda) y del ICD (derecha) en función del nivel de pericia de los futbolistas. Las barras de error representan el error estándar.

Relaciones entre el CETD y el ICD.

Se encontró una relación directa entre la competencia decisional global y la inteligencia contextual en el deporte, tal y como confirmó la prueba de correlación bivariada de Pearson entre las puntuaciones globales del CETD y del ICD, $(r=.57, p<.001)$. Se puede observar cómo la Competencia Decisional Percibida y el Compromiso con el Aprendizaje Decisional se relacionan de forma positiva con la Inteligencia Anticipatoria, Inteligencia Táctica e Inteligencia Competitiva, mientras que, como cabría esperar, la Ansiedad y Agobio al Decidir se relaciona de forma negativa con las tres dimensiones de la inteligencia contextual. Si estas relaciones las analizamos en función del nivel de los jugadores encontramos que las relaciones varían de un nivel a otro (Tabla 1). Los resultados muestran que, al contrario que los futbolistas de nivel autonómico y nacional, los jugadores internacionales no muestran una relación significativa entre la Ansiedad al Decidir y las subescalas del Cuestionario de Inteligencia Contextual. Resulta llamativo que las relaciones entre la Competencia Decisional Percibida y la Inteligencia Táctica en los jugadores de categoría nacional sea negativa, cuando en el resto y en terminos globales es todo lo contrario. Del mismo modo, la relación entre la Inteligencia Táctica y el Compromiso con el Aprendizaje fue menor a medida que aumentaba el nivel de pericia, llegando a no relacionarse en el caso de los internacionales.

Tabla 1. Correlaciones entre las diferentes escalas del CETD y del ICD en función del nivel de pericia de los futbolistas.

\begin{tabular}{lccc}
\hline & $\begin{array}{c}\text { Inteligencia } \\
\text { Anticipatoria }\end{array}$ & Inteligencia Táctica & $\begin{array}{c}\text { Inteligencia } \\
\text { Competitiva }\end{array}$ \\
\hline Competencia Decisional Percibida & $.48^{* * *}$ & $.65^{* * *}$ & $.55^{* * *}$ \\
Autonómico & $.28^{* * *}$ & $-45^{* * *}$ & $.20^{* * *}$ \\
Nacional & $.47^{* * *}$ & $.66^{* * *}$ & $.41^{* * *}$ \\
Internacional & $.37^{* * *}$ & $.55^{* * *}$ & $.41^{* * *}$ \\
Total & & & \\
Ansiedad y Agobio al Decidir & $-.25^{* * *}$ & $-.31^{* * *}$ & $-.38^{* * *}$ \\
Autonómico & $-.18^{* * *}$ & $-.24^{* * *}$ & $-.21^{* * *}$ \\
Nacional & -.09 & -.05 & -.04 \\
Internacional & $-.20^{* * *}$ & $-.25^{* * *}$ & $-.24^{* * *}$ \\
Total & & & $.42^{* * *}$ \\
Compromiso en el Aprendizaje & $.45^{* * *}$ & $.32^{* * *}$ & $.41^{* * *}$ \\
Decisional & $.30^{* * *}$ & $.13^{*}$ & $.33^{* *}$ \\
Autonómico & $.32^{* *}$ & .05 & $.40^{* * *}$ \\
Nacional & $.35^{* * *}$ & $.19^{* * *}$ & \\
Internacional & & & \\
Total &
\end{tabular}

Nota: $* * * p<.001, * * p<.01, * p<.05$ 
Ruiz-Pérez, L. M.; Navia, J. A.; Miñano-Espín, J.; Garcia-Coll, V. y Palomo-Nieto, M. (2015). Autopercepción de inteligencia contextual para jugar y de competencia decisional en el fútbol. RICYDE. Revista internacional de ciencias del deporte, 42(11), 329-338. http://dx.doi.org/10.5232/ricyde2015.04202

\section{Discusión}

El presente estudio tuvo como objetivo analizar las relaciones entre las autopercepciones de inteligencia contextual y de competencia para tomar decisiones en jugadores de fútbol de diferente nivel de rendimiento. Los resultados mostraron que los participantes en este estudio presentaron mejoras en sus puntuaciones en los dos instrumentos a medida que aumentaba la categoría en la que participaban, lo que indica el aumento de dominio y competencia en el juego se relaciona con las autopercepciones que los jugadores tienen para resolver los problemas del juego (Baker, Coté y Abernethy, 2003; Graupera, Ruiz, García y Smith, 2011; Ruiz, García, Palomo, Navia y Miñano, 2014). La relación entre el nivel de rendimiento y las percepciones de competencia ha sido destacada por numerosos investigadores, así Deci y Ryan (1995) o el propio Bandura (1997) ya indicaron que los individuos que se perciben más competentes en un campo de actuación están más intrínsecamente motivados a persistir y rendir en el mismo. Ser competente y percibirse competente son las dos dimensiones que interactúan en el rendimiento deportivo que la práctica y el entrenamiento tienen la obligación de promover y mantener (Ommundsen y Vaglum, 1997).

Se han podido comprobar las estrechas relaciones existentes entre las subescalas de los dos cuestionarios así como entre sus índices globales. Estas relaciones se pueden considerar más que moderadas por lo que incita pensar que están explorando dimensiones cognitivas y emocionales en las que subyace un factor general, un factor relacionado con la competencia para solucionar problemas que reclama comportarse tácticamente y que respondería a las intuiciones que entrenadores y jugadores tienen sobre lo que consideran un rendimiento inteligente en el fútbol. Especialmente, parecen relaccionarse la competencia decisional percibida y la inteligencia táctica, lo cual tiene sentido en la medida en que las decisiones que se toman en el terreno de juego reclaman la confianza necesaria para que sean tomadas y puestas en acción.

No obstante han emergido algunas relaciones que reclaman un análisis más detenido como es el hecho de que entre los jugadores de categoría nacional las relaciones entre la perecpión de competencia para decidir y la inteligencia táctica fuera inversa y de intensidad moderada $(\mathrm{r}=$ -.45), lo que nos indicaría que para estos jugadores, cuanto más aumenta su percepción de inteligencia para solucionar los problemas tácticos del juego, sin embargo disminuye su confianza para tomar las decisiones necesarias para ponerlos en acción, algo que cambia radicalmente en el caso de los internacionales $(r=.60)$.

Es razonable pensar que si un deportista se considera que posee una buena inteligencia táctica para su deporte también se perciba competente para decidir en el mismo, como se constata entre los deportistas de nivel internacional sin embargo, no siempre acompaña a los deportistas grandes dosis de confianza. El deporte de alto rendimiento está asociado a altos niveles de presión, lo que supone mayores niveles de ansiedad, como se manifiesta en las relaciones entre los diferentes tipos de inteigencia y la dimensión ansiedad del CETD en los niveles nacional y autonómico. En comparación con el internacional, y como Woodman y Hardy (2003) indicaron, se esperaría una clara relación entre la confianza y el rendimiento en los deportistas de mayor nivel de pericia, como es el caso que nos ocupa.

La capacidad para actuar de manera inteligente y tomar las decisiones apropiadas está siempre sometida a los vaivenes de la disposición psicológica de los jugadores, a su resistencia mental para afrontar los retos (Hatfield y Kerick, 2007; Jones, 2012), y como cabría esperar es algo que se aprende, y que los jugadores de mayor nivel y mayor experiencia, muestran de forma más clara que los jugadores de niveles inferiores (Baker, Coté y Abernethy, 2003; Wein, 1995, 2005). 
Ruiz-Pérez, L. M.; Navia, J. A.; Miñano-Espín, J.; Garcia-Coll, V. y Palomo-Nieto, M. (2015). Autopercepción de inteligencia contextual para jugar y de competencia decisional en el fútbol. RICYDE. Revista internacional de ciencias del deporte, 42(11), 329-338. http://dx.doi.org/10.5232/ricyde2015.04202

En resumen, el empleo de cuestionarios como los utilizados en este estudio permiten al entrenador o al psicólogo del deporte, conocer lo que los jugadores piensan de sus propias competencias para desenvolverse en el juego. Estas percepciones son dinamizadoras de su deseo de practicar y de competir y su estudio se hace a todas luces necesario. No obstante, este estudio presenta la limitación de que todos sus participantes han sido varones, lo que abre la necesidad de estudiar estas dimensiones cognitivo-emocionales en las jugadoras de fútbol de diferentes categorías.

\section{Referencias}

Arnau, J. (1995). Psicología experimental: un enfoque metodológico. México: Trillas.

Baker, J.; Coté, J., \& Abernethy, B. (2003). Sport specific practice and the development of expert decision making in team ball sports. Journal of Applied Sport Psychology, $15,12-25$.

http://dx.doi.org/10.1080/10413200305400

Bandura, A. (1997). Self-efficacy: the exercise of control. New York: W. H. Freeman and Company.

Cianciolo, A. T.; Matthew, C.; Sternberg, R. J., \& Wagner, R. K. (2006). Tacit knowledge, practical intelligence and expertise. En K.A. Ericsson, N. Charness, P.J. Feltovich, y R.R. Hoffman (Eds.), The Cambridge Handbook of Expertise and Expert Performance (pp. 613-632). Cambridge: Cambridge University Press.

http://dx.doi.org/10.1017/cbo9780511816796.035

Deci, E. L., \& Ryan, R. M. (1985). Intrinsic motivation and self-determination in human behavior. New York: Plenum. http://dx.doi.org/10.1007/978-1-4899-2271-7

Drasgow, F. (2003). Intelligence and the workplace. En W. Borman, D. Ilgen y R. Klimoski (eds.), Handbook of Psychology, 12 (pp.107-129). New York: John Wiley.

Durán, J. P. (2003). El rendimiento experto en el deporte. Análisis de las variables cognitivas, psicosociales y de la práctica deliberada en lanzadores de martillo españoles de alto rendimiento. Tesis Doctoral Inédita. Universidad de Castilla - La Mancha, Toledo.

Elferink-Gemser, M. T.; Visscher, C.; Richard, H., \& Lemmink, K. A. (2005). Development of the tactical skills inventory for sports. Perceptual and Motor Skills, 99, 883-895. http://dx.doi.org/10.2466/pms.99.3.883-895

Furnham, A. (2005). Gender and personality differences in self and other ratings of business intelligence. British Journal of Management, 16, 91-103. http://dx.doi.org/10.1111/j.1467-8551.2005.00434.x

García, V.; Ruiz, L. M., y Graupera, J. L. (2009). Perfiles decisionales de jugadores y jugadoras de voleibol de diferente nivel de pericia. RICYDE.Revista Internacional de Ciencias del Deporte, 14(5), 123-137. http://dx.doi.org/10.5232/ricyde2009.01410

Gardner, H. (1983). Frames of mind: The theory of Multiple Intelligences. New Cork: Basic Books.

Gardner, H. (1987). Estructuras de la mente. La teoría de las múltiples inteligencias. México: Fondo de Cultura Económica. 
Ruiz-Pérez, L. M.; Navia, J. A.; Miñano-Espín, J.; Garcia-Coll, V. y Palomo-Nieto, M. (2015). Autopercepción de inteligencia contextual para jugar y de competencia decisional en el fútbol. RICYDE. Revista internacional de ciencias del deporte, 42(11), 329-338. http://dx.doi.org/10.5232/ricyde2015.04202

Gardner, H. (1993). Multiples intelligences: The theory in practice. New York: Basic Books.

Gardner, H. (2001). La inteligencia reformulada. Las inteligencias múltiples en el siglo $X X I$. Barcelona: Paidós.

Gaspar, P. (2001). Contributo para a validação transcultural do questionário estilo de tomada de decisão no desporto. Trabajo Fin de Master. Centro Olímpico de Estudios Superiores, Comité Olímpico Español, Madrid.

Gil, A.; Jiménez, R.; Moreno, M. P.; Moreno, B.; Del Villar, F., y García, L. (2010). Análisis de la motivación intrínseca a través de las necesidades psicológicas básicas y la dimensión subjetiva de la toma de decisiones en jugadores de voleibol. Revista Iberoamerica de Psicología del Ejercicio y el Deporte, 5,1, 29-44.

Gil. A.; Claver, F.; Alías, P.; Perla, M.; García-González, L., \& Del Villar, F. (2012). Influencia del rendimiento sobre el estilo decisional en jugadores de fútbol. Movimiento Humano, 3, 35-44.

Goleman, D. (1996). Inteligencia Emocional. Barcelona: Kairós.

Goleman, D. (2006). Inteligencia social. La nueva ciencia de las relaciones humanas. Barcelona: Kairós.

Goleman, D., \& Cherniss, C. (2001). Inteligencia emocional en el trabajo. Barcelona: Kairós.

Graupera, J. L.; Ruiz, L.M.; García, V. \& Smith, R. (2011). Development and validation of a Spanish version of an athletic coping skills inventory, ASCI-28. Psicothema, 23, 3, 495-502.

Griffith, C.R. (1928). Psychology and Athletics. New York: Scribners.

Hatfield, B. D., \& Kerick, S. E. (2007). The psychology of superior sport performance: A cognitive and affective neuroscience perspective. En G. Tenenbaum y R.C. Eklund (Eds.), Handbook of Sport Psychology (3rd Edition) (pp 84-112). New Jersey: John Wilet \& sons.

http://dx.doi.org/10.1002/9781118270011.ch4

Jiménez, C. (2004). Análisis de la toma de decisiones en los deportes colectivos: estrategias de las jugadoras aleros de baloncesto en posesión de balón. Tesis doctoral inédita. Universidad de Castilla - La Mancha, Toledo.

Jones, G. (2012). The role of Superior Performence Intelligence in sustained success. En S.M. Murphy (Ed.), The Oxford Handbook of Sport and Performance Psychology (pp. 62-80). Oxford: Oxford University Press. http://dx.doi.org/10.1093/oxfordhb/9780199731763.013.0004

Jones, J. M. (2012). Emotion regulation and performance. En S.M. Murphy (Ed.), The Oxford Handbook of Sport and Performance Psychology (pp. 154-172). Oxford: Oxford University Press. http://dx.doi.org/10.1093/oxfordhb/9780199731763.013.0008

López, J. (2002). Estudio de la toma de decisión en baloncesto de silla de ruedas. Trabajo Fin de Master. Master Europeo en Discapacidades UNED. Madrid.

Navia, J. A. (2013). Estrategias visuales y motrices de los porteros de fútbol sala en tareas de interceptación bajo presión temporal y espacial. Tesis Doctoral inédita. Universidad Politécnica de Madrid.

http://dx.doi.org/10.1080/00336297.1994.10484123 
Ruiz-Pérez, L. M.; Navia, J. A.; Miñano-Espín, J.; Garcia-Coll, V. y Palomo-Nieto, M. (2015). Autopercepción de inteligencia contextual para jugar y de competencia decisional en el fútbol. RICYDE. Revista internacional de ciencias del deporte, 42(11), 329-338. http://dx.doi.org/10.5232/ricyde2015.04202

Navia, J.A. y Ruiz, L.M. (2014). Análisis de la complejidad perceptivo-motriz y psicológica del penalti en el fútbol. RICYDE. Revista internacional de ciencias del deporte, $37(10), 264-280$.

http://dx.doi.org/10.5232/ricyde2014.03706

McPherson, S. (1994). The development of sport expertise: mapping the tactical domain. Quest, 46, 223-240.

Miranda, C. (2010). Estudio comparativo del perfil decisional en jugadores de voleibol en formación. Tesis de Master. Facultad de Ciencias de la Actividad Física y el Deporte. Universidad de Granada.

Ommundsen, Y., \& Vaglum, P. (1997). Competence, perceived competence and drop-out from soccer: a study of young playeres. Scandinavian Journal of Medecine \& Science in Sports, 7, 6, 373-383.

http://dx.doi.org/10.1111/j.1600-0838.1997.tb00170.x

Peñaloza, R. (2007). Estudio y análisis del comportamiento visual de deportistas de taekwondo con diferente nivel de pericia. Tesis Doctoral inédita. Universidad CastillaLa Mancha, Toledo.

Ruiz, L. M.; Graupera, J. L., y Navarro, F. (1998). Construcción, análisis psicométrico y tipificación de un cuestionario de estilos de toma de decisión en el deporte. CARID: Consejo Superior de Deportes.

Ruiz, L. M.; Graupera, J. L., y Sánchez, F. (2000). Dimensión subjetiva de la toma de decisiones en el deporte. Desarrollo y validación del Cuestionario CETD de un cuestionario de estilo de decisión en el deporte. CARID: Consejo Superior de Deportes.

Ruiz, L.M.; Durán, J. P.; Jiménez, C., y Sánchez (2006). Los expertos en el deporte: Su estudio y análisis desde una perspectiva psicológica. Anales de Psicología, 22, 132142.

Ruiz, L. M.; Durán, J., y Arruza, J. A. (2007). Rendimiento experto y percepción de las actividades de entrenamiento en lanzadores de martillo españoles del alto rendimiento. Anales de Psicología, 23, 2, 300-306.

Ruiz, L. M.; García, V.; Palomo, M.; Manzano, J. A., y Miñano, J. (2013). Inteligencia Contextual y perica en el fútbol. Revista Internacional de Medicina y Ciencias de la Actividad Física y el Deporte, 14, 307-317.

Ruiz, L. M.; Graupera, J. L., y García, V. (2014). Inteligencia Contextual Percibida en el Deporte. Desarrollo y Validación de un cuestionario. Cultura, Ciencia y Deporte 9, 211-224.

Ruiz, L. M.; Palomo, P.; García, V.; Navia, J. A., Miñano, J., \& Psotta, R. (2014). Selfperceptions of decision making competence in Spanish football players. Acta Universitatis Palackianae Olomucensis. Gymnica, 44, 2. 77-83

Sternberg, R. J. (2000). Intelligence and wisdom. En R. Sternberg (Ed.), Handbook of Intelligence (pp. 631-649). New York: Cambridge University Press. http://dx.doi.org/10.1017/cbo9780511807947.029

Sternberg, R. J.; Forsythe, G. B.; Hedlund, J.; Horvath, J. A.; Wagner, R. K.; Williams, W. M.; Snook, S. A., \& Grigorenko, E. L. (2000). Practical Intelligence in everyday life. Cambridge: Cambridge University Press.

Tenenbaum, G. (2009). The study of perception: From the perceptions of traits and states to the perception of effort, and the perceptual-cognitive-emotion-motor linkage. Proceedings of the 12th ISSP World Congress of Sport Psychology (p.12). Marrakech, Morocco. 
Ruiz-Pérez, L. M.; Navia, J. A.; Miñano-Espín, J.; Garcia-Coll, V. y Palomo-Nieto, M. (2015). Autopercepción de inteligencia contextual para jugar y de competencia decisional en el fútbol. RICYDE. Revista internacional de ciencias del deporte, 42(11), 329-338. http://dx.doi.org/10.5232/ricyde2015.04202

Tennenbaum, G., \& Bar-Eli, M. (2007). Personality and intellectual capabilities in sport psychology. En D. Smith y M. Bar Eli (Eds.), Essential readings in sport and exercise psychology (pp.102-120). Champaign: Human Kinetics.

Terenzini, P. T. (1993). On the nature of Institutional Research and the knowledge and skills it requires. Research in Higher Education, 34, 1, 1-10. http://dx.doi.org/10.1007/BF00991859

Wagner, R. K. (1987). Tacit knowledge in everyday intelligent behaviour. Journal of Personality and Social Psychology, 52, 1236-1247. http://dx.doi.org/10.1037/0022-3514.52.6.1236

Woodman, T. \& Hardy, L. (2003). The relative impact of cognitive anxiety and selfconfidence upon sport performance: a meta-analysis. Journal of Sport Sciences, 21, 443-457.

http://dx.doi.org/10.1080/0264041031000101809 